treatment $(\mathrm{p} \leq 0.0005)$. With this limited sample, clinical impact at 6 months was not significant.

No conflict of interest.

\section{OHP-046 IMPACT OF MULTI-LUMEN INFUSION DEVICES ON THE OCCURRENCE OF PHYSICAL DRUG INCOMPATIBILITIES: A CONTROLLED IN VITRO STUDY}

doi:10.1136/ejhpharm-2013-000276.420

'M Perez, ' ${ }^{1} \mathrm{~A}$ Foinard, ${ }^{1} \mathrm{C}$ Barthélémy, ${ }^{2} \mathrm{~B}$ Debaene, 'B Décaudin, 'P Odou. 'Faculté de Pharmacie, Laboratoire de Biopharmacie Pharmacie Galénique et Hospitalière, Lille Cedex, France; ${ }^{2} \mathrm{CHU}$ de Poitiers, Département d'Anesthésie, Poitiers, France

Background Drug incompatibility is a problem when managing patients in intensive care units. Patients receive many drugs simultaneously but through limited venous accesses. The recent marketing of new multi-lumen infusion access device may open the way to preventing incompatibility.

Purpose To evaluate the impact of multi-lumen infusion access devices connected to single-lumen central venous catheters on the occurrence of known drug incompatibilities through a controlled in vitro study.

Materials and Methods Two infusion devices were studied: 1) a standard set with six-gang-manifolds and its extension line and 2) a multi-lumen infusion access device with nine lumens (Edelvaiss-Multiline, Doran International, France). Six drugs were selected: three basic drugs (furosemide, pantoprazole and amoxicillin/clavulanic acid) and three acid drugs (amiodarone, dobutamine and midazolam). Two, four or six drugs and an infusion vehicle (saline, Ringer's or 5\% glucose) were infused simultaneously. The infusion rate of the vehicle was initially set at $100 \mathrm{~mL} / \mathrm{h}$ and decreased stepwise by $10 \mathrm{~mL} / \mathrm{h}$ until precipitate formation occurred. Physical incompatibility was assessed by visual inspection and sub-visible particle count test as defined by the European Pharmacopeia according to the European Pharmacopeia. The lowest value of the vehicle infusion rate that satisfied the two tests was reported for each infusion set and for each drug combination.

Results The use of multiline access devices contributed to preventing drug incompatibilities when simultaneously infusing two and four drugs. Indeed, infusion vehicle flow rate gains oscillated between 10 and $40 \mathrm{~mL}$ per hour, in more than $55 \%$ and $25 \%$ of cases, respectively for two and four drugs. When infusing six drugs simultaneously, no differences were identified.

Conclusions Our main hypothesis is that fluid dynamics differ depending on the infusion device resulting in differences in the contact time between drugs. Under specified infusion conditions, the nine-lumen device prevents physical drug incompatibilities.

No conflict of interest.

\section{OHP-047 IMPACT OF THE ECONOMIC CRISIS ON BIOMEDICAL RESEARCH: ANALYSIS OF THE WORK OF A CLINICAL RESEARCH ETHICS COMMITTEE}

doi:10.1136/ejhpharm-2013-000276.421

P Arcenillas, S Redondo, N Giménez, P March, L Soriano, R Pla, S Quintana. Hospital Universitari Mútua de Terrassa, Pharmacy, Terrassa, Spain

Background Since 2008, the economic crisis has directly affected many activities, health and biomedical research being particular fields involved.

Purpose To evaluate the impact of the economic crisis on research at a Spanish hospital based on an analysis on the work done by the Clinical Research Ethics Committee (CEIC). To compare it with any effects on Spanish and European CTs.
Materials and Methods CEIC Minutes from a 500-bed university hospital were reviewed from 2000 to 2011, obtaining information from clinical trials (CTs) and observational studies (OS).

The financing of CTs was classified: 1) CTs promoted by the pharmaceutical industry, 2) by scientific societies with industry support, 3) by scientific societies with government support and 4) unfunded CTs. We compared two periods: pre-crisis (2000-2007) and crisis (2008-2011).

National scientific activity was obtained from a secondary data source from the information provided by the Agencia Española de Medicamentos y Productos Sanitarios and the European activity from EU Clinical Trials Registers (European Medicines Agency website).

Data analysis used conventional descriptive statistics.

Results 782 protocols were evaluated (average 71 protocols/year).

During 2008-2011 there was an annual average decrease of 13 CT in groups 1 and 2, compared with the period 2000-2007 (95\% CI: 4-22 CT).

Regarding the OSs, there was an annual average increase of 36 OSs during the second period (95\% CI: $24-49$ OS). There were no statistical differences between the two periods for groups 3 and 4.

The total number of protocols increased by an average of 25 projects/year during the second period compared to the first $(95 \% \mathrm{CI}$ : $8-40$ projects).

There were 2340 CTs in Spain during the first period and 3096 during the second $(p=n s)$. CTs in Europe were 7,908 and 10,632 respectively $(p=n s)$.

Conclusions The CEIC workload was maintained, even increased, but because of OSs and unfunded research. The crisis marked a turning point; funded studies decreased and OSs increased.

At the moment there are no noteworthy changes in Spanish or European CT activity.

Abstract OHP-047 Table 1

\begin{tabular}{lll}
\hline & Number of CTs & $\%$ \\
\hline Group 1 & 407 & 52 \\
Group 2 & 53 & 7 \\
Group 3 & 32 & 4 \\
Group 4 & 93 & 12 \\
OS & 197 & 25 \\
\hline * OS were analysed globally
\end{tabular}

No conflict of interest.

\section{OHP-048 IMPLEMENTATION OF A PROTOCOL FOR SELECTION OF BIOLOGICAL THERAPIES IN RHEUMATOLOGY}

doi:10.1136/ejhpharm-2013-000276.422

YM Alonso-Triana, H Navarro, R Huarte, I Larrodé, M Sebastián-Aldeanueva, MJ Perrino, R Abad. Servet, Pharmacy, Zaragoza, Spain

Background The Public Health System in our Autonomous Community has established a protocol for biological treatments (BTs) in rheumatoid arthritis (RA), spondyloarthropathies (SAPs) and juvenile idiopathic arthritis (JIA).

Purpose To evaluate the implementation of the BTs protocol and to analyse the use of these treatments.

Materials and Methods We analysed patients who had started treatment with BT or been switched from a previous biological treatment, since the implementation of the protocol (12/05/2011 to 29/02/2012). This document has different levels of decision based on both disease status and treatment effectiveness; RA: 1st level: infliximab or subcutaneous tumour necrosis factor inhibitor (anti-TNF) (etanercept or adalimumab); 2nd: tocilizumab or abatacept or rituximab; 3rd: golimumab or certolizumab pegol. SAPs: 1st level: infliximab or etanercept or adalimumab; 2 nd: golimumab; 3 rd: infliximab 